\title{
Anatomical variants and coronary anomalies detected by dual-source coronary computed tomography angiography in North-eastern Thailand
}

\author{
Narumol Chaosuwannakit \\ Radiology Department, Faculty of Medicine, Khon Kaen University, Khon Kaen, Thailand
}

\begin{abstract}
Purpose: Congenital coronary anomalies are uncommon, with an incidence ranging from $0.17 \%$ in autopsy cases to $1.2 \%$ in angiographically evaluated cases. The recent development of dual-source coronary computed tomography angiography (coronary CTA) allows accurate and noninvasive depiction of coronary artery anomalies.

Material and methods: A retrospective study included a total of 924 patients who underwent coronary CTA because of known or suspected coronary artery disease. In each study, coronary artery anomalies (CAs) were investigated.

Results: A total of 924 patients (mean age $51.2 \pm 12.8$ years), who underwent dual-source coronary CTA, were studied. The overall prevalence of CAs in our study was $3.7 \%$, with the following distribution: four single coronary artery, 14 anomalous origin from opposite sinus of Valsalva, three absent left main, four high take-off coronary artery, three anomalous left coronary artery from pulmonary artery, and eight coronary artery fistulas.

Conclusions: The present study supports the use of coronary CTA as a reliable noninvasive tool for defining anomalous coronary arteries in an appropriate clinical setting and provides detailed three-dimensional anatomic information that may be difficult to obtain with invasive coronary angiography.
\end{abstract}

Key words: anomalous coronary artery, prevalence, coronary CTA.

\section{Introduction}

Coronary artery anomalies (CAs) are uncommon, with a prevalence of $1 \%$, but figures between 0.3 and $5.6 \%$ have been reported in the literature [1-4]. For several decades, the diagnosis of CAs is usually established by invasive coronary angiography (ICA). However, due to the two-dimensional projection nature of ICA, the visualisation of a complex three-dimensional vessel course as well as clarification of the exact relationship with surrounding anatomical structures may be difficult, and misinterpretation is reported in up to $50 \%$ of cases [1,5]. Recently, coronary computed tomography angiography (CTA) has emerged as a noninvasive alternative for the evaluation of CAs [6]. Recently developed computed tomography scanners with 128 -slice detectors are able to achieve an improved temporal resolution of up to $83 \mathrm{~ms}$. It is now possible and practical to have high-quality cardiac imaging without $\beta$-blocker premedication, even in patients with high heart rates, particularly after the introduction of modern protocols $[7,8]$ that allowed coronary CTA with a radiation dose substantially lower than that of ICA [9]. Therefore, coronary CTA has been recommended as the first-line method for the assessment of known or suspected CAs [10]. Most of the CAs have been detected incidentally during the evaluation of patients with suspected coronary artery disease. Although CAs lack clinical significance in the majority of these patients, certain abnormal patterns, such as the anomalous origin of a coronary vessel from the opposite sinus, have been as-

Correspondence address:

Narumol Chaosuwannakit, MD, FSCMR, Radiology Department, Faculty of Medicine, Khon Kaen University, Khon Kaen, Thailand, 40000,

e-mail: narumol_chao@yahoo.com

Authors' contribution:

A Study design - B Data collection - C Statistical analysis - D Data interpretation - E Manuscript preparation · F Literature search · G Funds collection 
sociated with sudden cardiac death and ischaemic complications $[2,3,5]$. In $17 \%$ of deaths of athletes, $12 \%$ of sport-related deaths in 14- to 40-year-old individuals are due to CAs [3,4]. As far as we know, this is the first study to determine the prevalence and describe the coronary artery anomalies in referred patients undergoing coronary CTA by dual-source CT in North-eastern Thailand.

\section{Material and methods}

\section{Patient population}

This retrospective study included 924 patients who underwent coronary CTA between December 2014 and December 2017. The indications for coronary CTA were an abnormal, equivocal, or non-diagnostic stress test, atypical chest pain, patients awaiting valvular surgery to detect or exclude associated coronary stenoses, suspected anomalous coronary, complex congenital heart disease, as well as the evaluation of cardiac aetiology of syncope. The above are considered appropriate indications for coronary CTA based on the criteria of the American College of Cardiology (ACC) [10]. Exclusion criteria for coronary CTA included the presence of multiple ectopic beats, atrial fibrillation, pregnancy, renal failure, a history of allergic reaction to iodine-containing contrast agents, and the patients who underwent prior coronary stents or bypass graft surgery. The present study was approved by the Ethics Committee of the Faculty of Medicine, Khon Kaen University, Khon Kaen, Thailand.

\section{Coronary computed tomography angiography scanning protocol}

Coronary CTA examinations were performed on a dual-source CT scanner (Definition FLASH, Siemens Healthcare, Forchheim, Germany). The system is equipped with

Table 1. Prevalence of coronary artery anomalies (CAs), $n$ (\%)

\begin{tabular}{|l|c|}
\hline Anomalies of origin and course & $n(\%)$ \\
\hline Absent left main & $3(0.3)$ \\
\hline High take-off & $4(0.4)$ \\
\hline $\begin{array}{l}\text { Anomalous left coronary artery from pulmonary } \\
\text { artery (ALCAPA) }\end{array}$ & $3(0.3)$ \\
\hline \begin{tabular}{l} 
Anomalous origin from the opposite sinus \\
\hline RCA originating from the left coronary sinus
\end{tabular} & $12(1.2)$ \\
\hline LCA originating from the right coronary sinus & $2(0.2)$ \\
\hline Single coronary artery & $4(0.4)$ \\
\hline \begin{tabular}{l} 
Anomalies of intrinsic coronary arterial anatomy \\
\hline Myocardial bridging
\end{tabular} & $22(2.4)$ \\
\hline $\begin{array}{l}\text { Anomalies of coronary termination } \\
\text { Coronary artery fistulas }\end{array}$ & $8(0.9)$ \\
\hline
\end{tabular}

two X-ray tubes and two corresponding detectors mounted on a single gantry with an angular offset of $90^{\circ}$. The coronary CTA used automatic tube current modulation in $\mathrm{x}, \mathrm{y}$, and $\mathrm{z}$ directions (Care Dose 4D, Siemens Healthcare). The scanner technology enables a prospectively ECG-triggered high-pitch spiral acquisition (FLASH Spiral Cardio, Siemens Healthcare, Forchheim, Germany). The coronary CTA scan parameters were as follows: two X-ray sources, detector collimation $32 \times 0.6 \mathrm{~mm}$ with double sampling by rapid alteration of the focal spot in the longitudinal direction (Z-flying focal spot), rotation time $330 \mathrm{~ms}$, tube voltage $120 \mathrm{kV}$. Image acquisition was performed during inspiratory breath-hold. To familiarise the patient with the protocol, breath-holding was practiced before the examination. The scan was followed by a test bolus injection to calculate the peak of contrast enhancement time. Then the final coronary angiogram was taken. A bolus of iodinated contrast material (350 mg/ml, Omnipaque; GE Healthcare) at a dose of $1.5 \mathrm{ml} / \mathrm{kg}$ with dual-head power injector followed by $10-20 \mathrm{ml}$ of saline flush at a same rate to that of the contrast injection. Axial images were reconstructed with $0.75 \mathrm{~mm}$ slice thickness and $0.5 \mathrm{~mm}$ increment using a medium sharp convolution kernel (B26) and retrospective ECG gating. The reconstructions were performed in $5 \%$ steps over the entire R-R cycle using a single-segment algorithm that utilised quarter segments of projection data from both detectors. Patients were scanned in the supine position.

\section{Coronary computed tomography angiography image analysis}

All acquired coronary CTA images were transferred to a dedicated 3D-postprocessing workstation. Coronary CTA image analysis was performed by one cardiovascular and thoracic radiologist and blinded to the clinical data. An experienced radiologist evaluated the CAs by performing the maximum intensity projections (MIPs), curved multiplanar reformats (cMPRs), and volume rendering technique (VRT). The CAs were classified into anomalies of origin (number of coronary ostia, high take-off, ectopic coronary origin, anomalous origin from pulmonary artery), anomalies of course (myocardial bridging), and anomalies of termination (coronary artery fistula).

\section{Results}

In total, 924 patients were included (429 women and 495 men) with a mean age of $51.2 \pm 12.8$ years ( 3 days-91 years). The prevalence and type of anomalies of coronary arteries observed in this study population are outlined in Table 1 . The prevalence of coronary anomalies in the present study was $3.7 \%$ (excluded myocardial bridging).

In 693 cases (75\%) the right coronary artery (RCA) was dominant; in 120 cases (13\%) the left coronary artery 

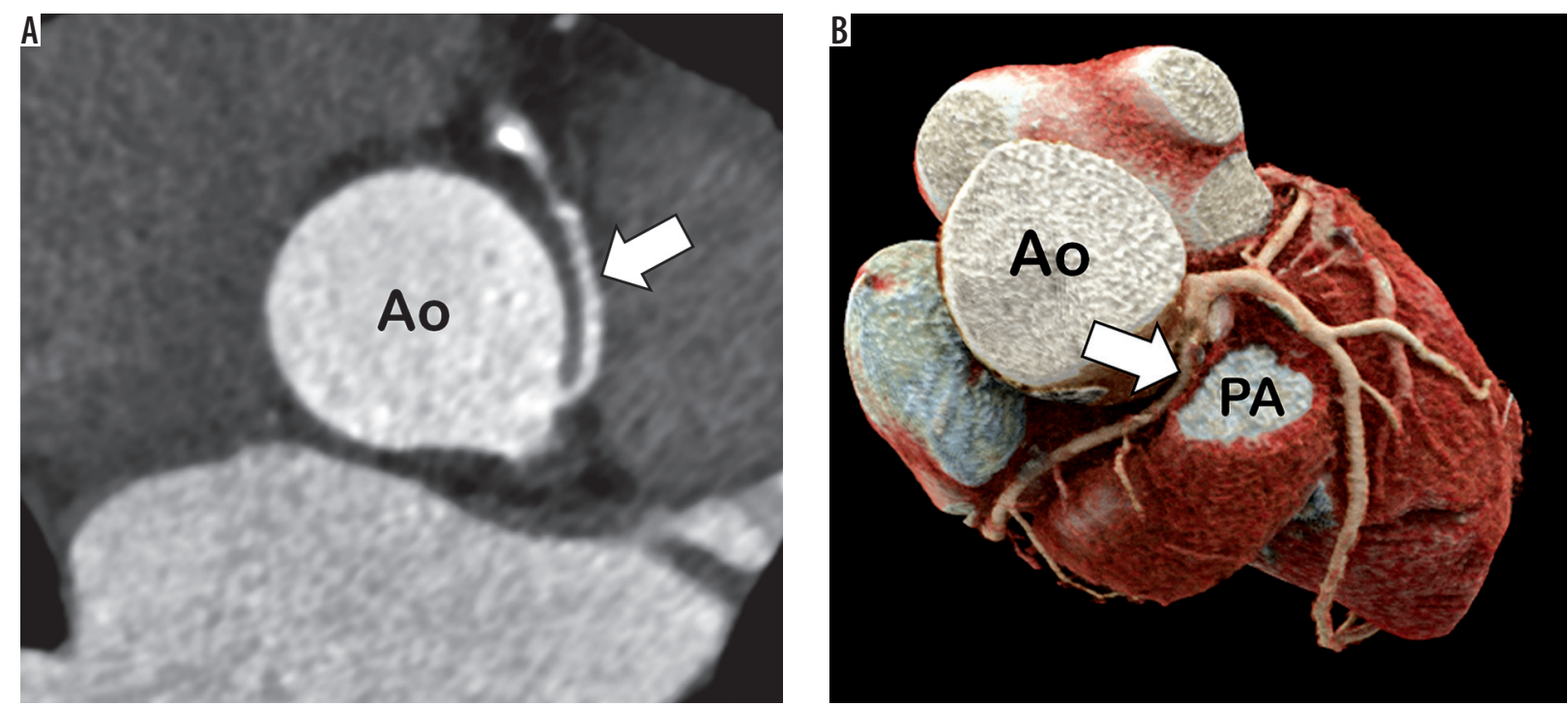

Figure 1. Images obtained in a patient with an anomalous origin of the right coronary artery (RCA) from the left sinus of Valsalva. Dual-source computed tomography coronary angiography axial view (A) and cinematic rendering technique (B) images showed the RCA originating from the left sinus of Valsalva (arrows) of the RCA, which is evident where the artery passes between the aortic root and the main pulmonary artery

Ao - aorta, PA - pulmonary artery, LAD - left anterior descending artery
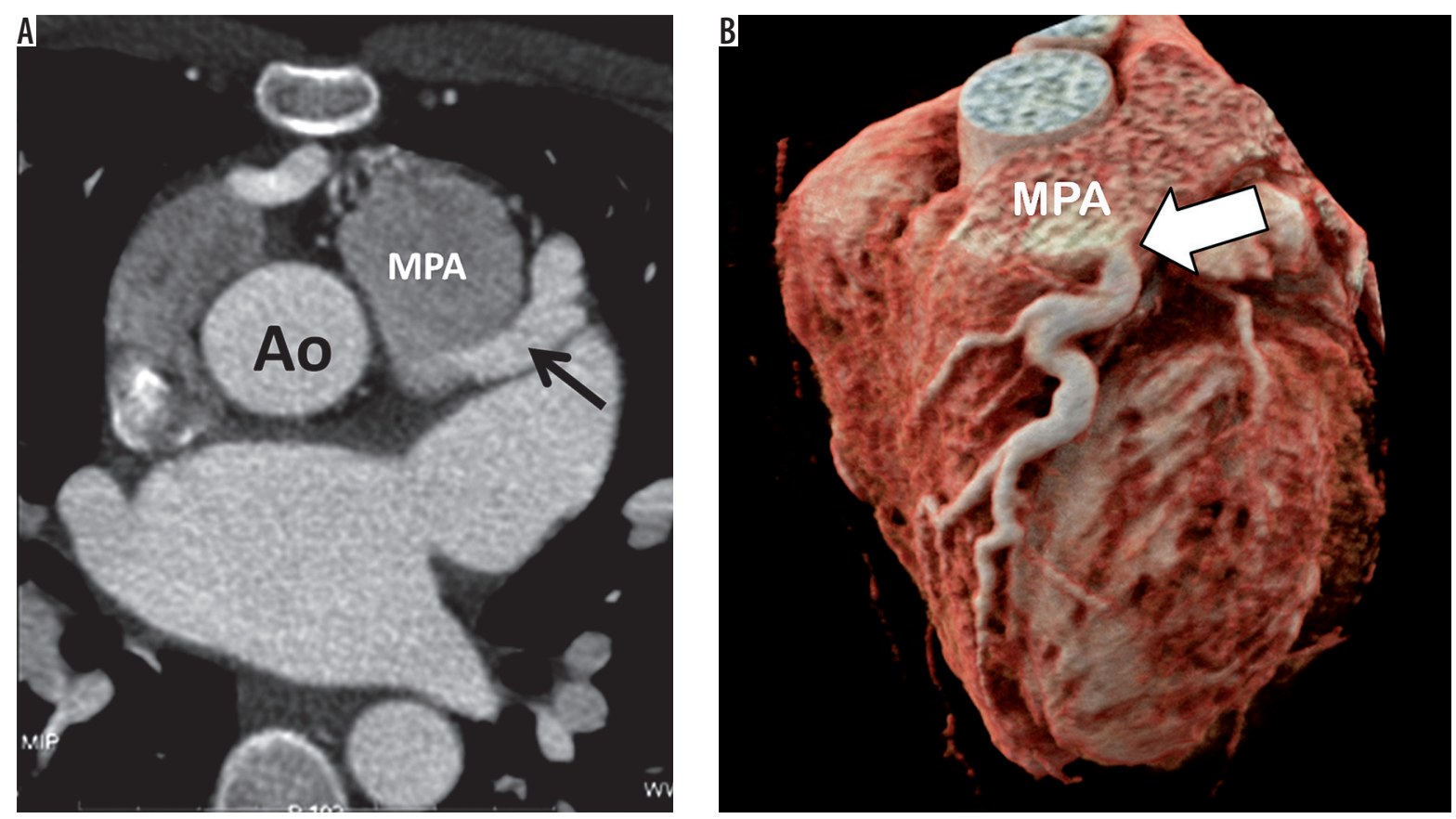

Figure 2. Dual-source computed tomography coronary angiography axial (A) and cinematic reconstruction (B) images showing anomalous left coronary artery ( $A$ and $B$; arrow) from main pulmonary artery (ALCAPA)

Ao - aorta, MPA - main pulmonary artery

(LCA) was dominant; and in 111 cases (12\%) co-dominance was observed. In 148 cases (16\%), the LCA trifurcated to include a third vessel between the left anterior descending artery (LAD) and circumflex $(\mathrm{Cx})$, termed ramus intermedius.

The left main coronary artery (LM) was absent in three cases $(0.3 \%)$ in which the left anterior descending artery (LAD) and left circumflex (LCx) originated from the left sinus of Valsalva with separate ostia. In 12 cases (1.2\%), the RCA originated from the left sinus of Valsalva: all had an interarterial course (Figure 1). In two cases $(0.2 \%)$, the LM originated from the right sinus of Valsalva: one had an interarterial course, and the other had a pre-pulmonic course. Three cases of right coronary artery with high takeoff origin and one case of left coronary artery with high take-off origin were recorded. In four cases (0.4\%), a single coronary ostium was found. In three cases, anomalous left coronary artery from pulmonary artery (ALCAPA) was 

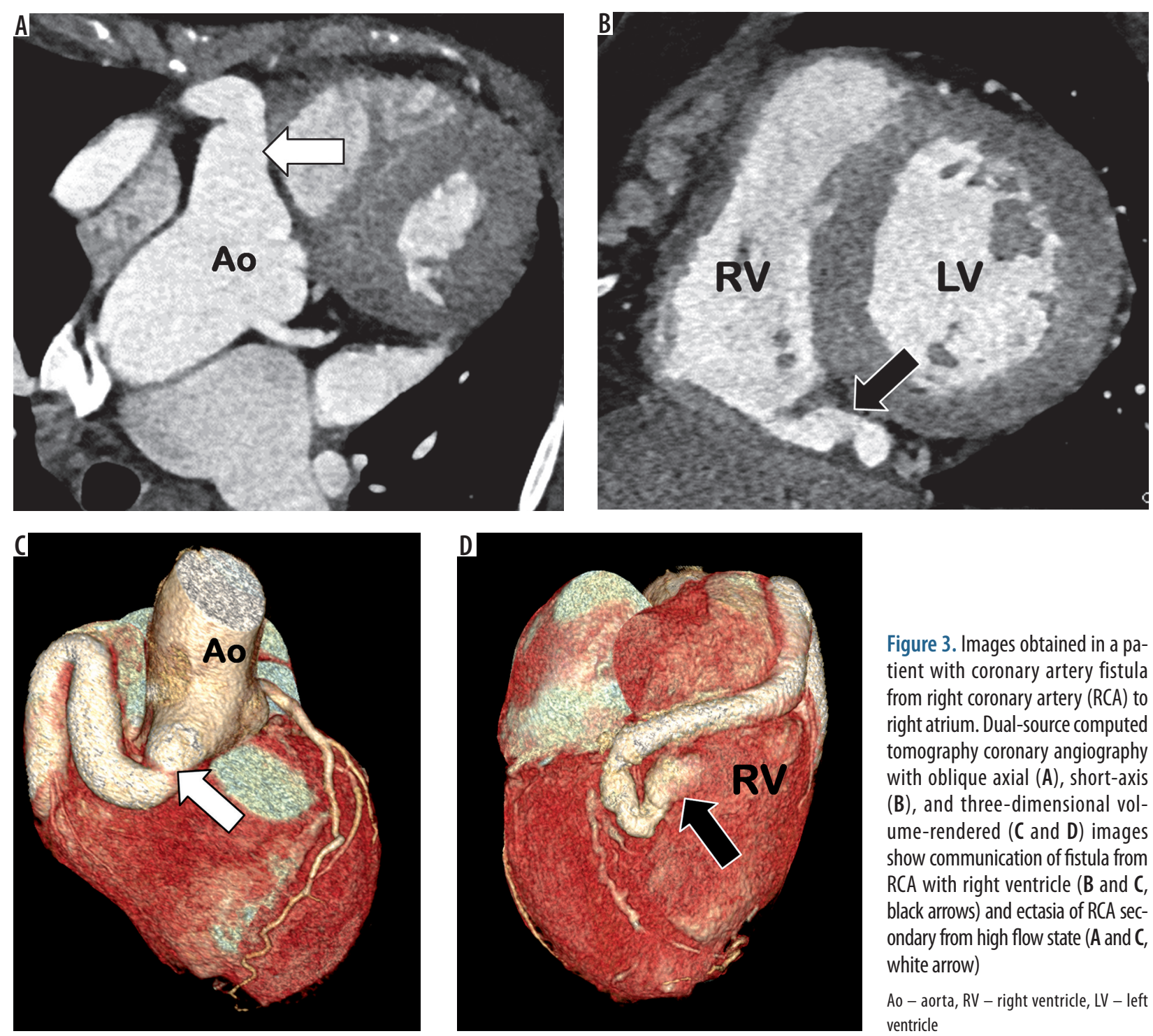

Figure 3. Images obtained in a patient with coronary artery fistula from right coronary artery (RCA) to right atrium. Dual-source computed tomography coronary angiography with oblique axial $(A)$, short-axis (B), and three-dimensional volume-rendered (C and $\mathbf{D})$ images show communication of fistula from RCA with right ventricle ( $B$ and $C$, black arrows) and ectasia of RCA secondary from high flow state (A and $C$, white arrow)

Ao - aorta, RV - right ventricle, LV - left ventricle

detected (Figure 2). Myocardial bridging was observed in 22 cases $(2.4 \%)$. All were identified at mid LAD. Eight cases $(0.9 \%)$ had fistulous tract connection branches from the RCA connected to the pulmonary artery (PA) or right ventricle (Figure 3).

\section{Discussion}

The incidence of anomalous coronary arteries in reported angiographic series ranges from $0.6 \%$ to $1.3 \%[1,2]$. Variations in the frequency of primary congenital coronary anomalies may possibly have a genetic background. Conventional ICA has been the technique of choice for visualisation of the coronary artery system for several decades. Despite its common use, alternative methods of visualising the coronary arterial system are desired; in addition to being invasive, ICA has disadvantages in detecting coronary artery anomalies because of the limited number of $2 \mathrm{D}$ projection images obtained during catheterisation and because of the absence of soft tissue information.
The overall prevalence of CAs in the present study is $3.7 \%$. Myocardial bridging was not included in the prevalence calculation because it was excluded from most of the previous studies in the literature. This percentage prevalence includes cases of anomalous origin and course as well as cases of anomalous termination. Although our results are similar to those of von Ziegler et al. [11], who reported a prevalence of $3.2 \%$, they are still high compared to other studies $[12,13]$. The high prevalence in this study may be attributed to the greater advances in imaging that made it less invasive and easier to image CAs accurately in the present study patient population. Another reason is that coronary CTA is considered as part of the preoperative assessment in congenital heart disease in our institution, which allowed us to examine a large number of congenital heart disease patients and, in turn, expanded the prospects of detecting coronary artery anomalies. Some classification systems of the CAs have been suggested $[3,14,15]$. According to these systems, anomalies of the CAs are divided into anomalies of origin and course, anomalies of intrinsic coronary arterial anatomy, 
and anomalies of termination. In our study, we used the anomalies of origin, course, and termination in addition to the CA variants. Some authors consider the multiple ostia or absent LM as normal variants [16], while others include it under the category of anomalous origin [17,18], as we did in the current study. We detected three cases $(0.3 \%)$ showing absence of left main. Instead, the LAD and LCX had separate ostia from the left sinus of Valsalva, which is in line with previous studies $[19,20]$. Clinically, it is important to recognise this anomaly to prevent complications in patients undergoing cardiac surgery, who require selective coronary cardioplegia such as aortic valve replacement [20]. Although the inability to separately cannulate the LCX or LAD may result in the erroneous assumption that the vessel is absent or occluded on invasive coronary angiography, the anatomic display of coronary CTA makes this diagnostic error uncommon $[18,20]$. An anomalous coronary artery can arise from the noncoronary sinus or the opposite sinus. The abnormal CA then takes one of the four following paths depending on the anatomic relationship of the anomalous vessel to the aorta and the pulmonary trunk. It may proceed into inter-arterial (between the aortic root and the pulmonary trunk), retro-aortic (dorsal to the aortic root), pre-pulmonic (anterior to the PA or right ventricular outflow tract), or trans-septal or sub-pulmonic (beneath the right ventricular outflow track) course [15]. The inter-arterial course is considered to be a malignant subtype, with a high risk of sudden cardiac death, especially when the LCA originates from the right coronary sinus. Theories about this anomaly's malignant potential include ischaemia from compression of the vessel by the great vessels at the interarterial component of the artery, acute take-off from the aortic wall, an associated ostial ridge, a slit-like ostium, intramural (inside the wall of the aortic root) course, superimposed spasm, intimal plaque, and clotting [21-25]. Although many patients are asymptomatic at the time of presentation or diagnosis, surgical correction is recommended due to the risk of ischaemic sudden death [21-25]. In our sample population, we observed nine cases of anomalous RCA origin from the left sinus of Valsalva and one case of anomalous LCA origin from the right sinus of Valsalva with inter-arterial course between the aortic root and PA. We had one case in which the left coronary artery originated from the right sinus of Valsalva with the trans-septal subtype. Single coronary artery is a situation in which the LCA and RCA arise with a common ostium from the right, left, or non-coronary sinus. Single coronary artery is among the rarest coronary artery anomalies, seen in only $0.024-0.044 \%$ of the population [26,27]. A single coronary artery is thought to be associated with a risk of sudden cardiac death, even in patients without a malignant arterial course. Demonstration of significant ischaemia in a symptomatic patient therefore warrants further clinical attention [26,27]. The single anomalous trunk supplies blood to the entire heart and is classified according to its origin, branching pattern, and course. In the current study, we had four $(0.4 \%)$ cases of single coronary artery, which is a higher prevalence than previously published. The presence of a single CA can be an isolated finding (one case in the present study) or associated with additional cardiac anomalies (three cases in the present study associated with complex congenital heart disease) [28]. As previously mentioned with the ectopic origin of the CA, the course taken by the vessels after the division of a single vessel to the right and left has greater clinical significance [29]. Myocardial bridging $(\mathrm{MB})$ is a congenital condition in which a segment of major epicardial CA proceeds intramurally through the myocardium beneath the muscle bridge [30]. The MB was the most frequently found anomaly, diagnosed in 18 cases $(2 \%)$ in the current study. Myocardial bridging is considered a benign condition that most commonly affects the middle segment of the LAD [30,31]. However, the relationship between MB anomaly and increased cardiovascular morbidity is still unclear. Coronary artery fistulas are present in $0.002 \%$ of the general population and are visualised in nearly $0.25 \%$ of patients undergoing cardiac catheterisation [32]. The majority of the fistulas drain into the PA or right ventricle, but fistulae may also drain into anywhere from between the vena cava or coronary sinus (CS) to the PA or left atrium (LA) [33]. Multiple fistulae may also occur. Over $90 \%$ of fistulae drain into the right side of the heart causing right to left shunt. Chronic large-volume shunts through these fistulae may cause major aneurysmal enlargement of the proximal feeding $\mathrm{CA}$ in addition to enlargement of the receiving vessel or chamber. The uninvolved CAs are usually normal [32]. A study by Zhou et al. [34] reported that fistulae more commonly (50.0\%) originate from both the LCA and the RCA followed by the LCA in $30.4 \%$ and RCA in $12.0 \%$. The remaining $7.6 \%$ of the cases were associated with extra-cardiac communications. In the current study, we encountered eight cases of coronary-pulmonary artery-cameral fistula (1\%). The diameter of the RCA was relatively increased because it is the primary feeder of the fistula. Clinical symptoms are proportional to the amount of induced haemodynamic abnormality. Patients with small CA fistulas remain asymptomatic, whereas those with high-flow fistulas may develop haemodynamic steal phenomenon with consequent myocardial ischaemia [35].

This study has several limitations that may artificially have raised the incidence of the anomalies reported. One limitation was the retrospective design of the trial, which was prone to confounding factors and bias. Moreover, the small sample size and the highly selected study population in our sample does not represent the true prevalence in a more general population because the cases were collected from a tertiary care referral medical school hospital.

The prevalence of CAs in the present study was 3.7\%, which is relatively higher than the average percentage reported in the literature. A further elaborate follow-up 
study is needed to analyse the exact prevalence of congenital coronary anomalies in the whole population of Thailand. Furthermore, the study included patients who underwent coronary CTA because of an anomaly that was seen or suspected in catheterisation. Surgical confirmation of coronary CTA findings was not available because not all of them underwent a surgical correction, especially those with benign anomalous origin and small fistulas.

\section{Conclusions}

The present study shows that coronary CTA is a robust noninvasive modality for delineating coronary artery anomalies. Complex anatomy of the coronary arteries in
3D can be accurately depicted by coronary CTA. Knowledge of CT appearances of various coronary artery anomalies and an understanding of the clinical significance of these anomalies are essential in making a correct diagnosis and planning patient treatment.

\section{Acknowledgements}

The authors would like to thank the Faculty of Medicine for its support.

\section{Conflict of interest}

The authors report no conflict of interest.

\section{References}

1. Kardos A, Babai L, Rudas L, et al. Epidemiology of congenital coronary artery anomalies: a coronary arteriography study on a central European population. Cathet Cardiovasc Diagn 1997; 42: 270-275.

2. Yamanaka O, Hobbs RE. Coronary artery anomalies in 126,595 patients undergoing coronary arteriography. Cathet Cardiovasc Diagn 1990; 21: 28-40.

3. Angelini P, Velasco JA, Flamm S. Coronary anomalies: incidence, pathophysiology, and clinical relevance. Circulation 2002; 105: 2449 2454.

4. Angelini P. Coronary artery anomalies: an entity in search of an identity. Circulation 2007; 115: 1296-1305.

5. Ishikawa T, Brandt PW. Anomalous origin of the left main coronary artery from the right anterior aortic sinus: angiographic definition of anomalous course. Am J Cardiol 1985; 55: 770-776.

6. Dhoble A, Dewar J, Szerlip M, Abidov A. Rare coronary anomaly: Common origin of major coronary arteries from the right sinus of Valsalva and a small ramus branch originating from the left sinus of Valsalva. Int J Cardiol 2012; 28: e3-4.

7. Ghadri JR, Kuest SM, Goetti R, et al. Image quality and radiation dose comparison of prospectively triggered low-dose CORONARY CTA: 128-slice dual-source high-pitch spiral versus 64-slice single-source sequential acquisition. Int J Cardiovasc Imaging 2012; 28: 1217-1225.

8. Achenbach S, Marwan M, Ropers D, et al. Coronary computed tomography angiography with a consistent dose below $1 \mathrm{mSv}$ using prospectively electrocardiogram-triggered high-pitch spiral acquisition. Eur Heart J 2010; 31: 340-346.

9. Herzog BA, Wyss CA, Husmann L, et al. First head-to head comparison of effective radiation dose from low-dose 64-slice CT with prospective ECG-triggering versus invasive coronary angiography. Heart 2009; 95: 1656-1661.

10. Taylor AJ, Cerqueira M, Hodgson JM, et al. ACCF/SCCT/ACR/ AHA/ASE/ASNC 2010 Appropriate Use Criteria for Cardiac Computed Tomography: a report of the American College of Cardiology/ Foundation Appropriate Use Criteria Task Force Society of Cardiovascular Computed Tomography/American College of Radiology/ American Heart Association/American Society of Echocardiogra-
phy/American Society of Nuclear Cardiology/Society for Cardiovascular Angiography and Interventions/Society for Cardiovascular Magnetic Resonance. J Am Coll Cardiol 2010; 56: 1864-1894.

11. von Ziegler F, Pilla M, McMullan L, et al. Visualization of anomalous origin and course of coronary arteries in 748 consecutive symptomatic patients by 64 -slice computed tomography angiography. BMC Cardiovasc Disord 2009; 11: 9-54.

12. Sundaram B, Kreml R, Patel S. Imaging of coronary artery anomalies. Radiol Clin North Am 2010; 48: 711-727.

13. Xu H, Zhu Y, Zhu X, et al. Anomalous coronary arteries: depiction at dual-source computed tomographic coronary angiography. J Thorac Cardiovasc Surg 2010; 143: 1286-1291.

14. Kacmaz F, Isiksalan N, Alyan O, et al. Imaging of coronary artery anomalies: the role of multidetector computed tomography. Coron Artery Dis 2008; 19: 203-209.

15. Kim SY, Seo JB, Do KH, et al. Coronary artery anomalies: classification and ECG-gated multi-detector row CT findings with angiographic correlation. Radiographics 2006; 26: 317-333.

16. Young PM, Gerber TC, Williamson EE, et al. Cardiac imaging: part 2, normal, variant, and anomalous configurations of the coronary vasculature. AJR Am J Roentgenol 2011; 197: 816-826.

17. Namgung J, Kim JA. The prevalence of coronary anomalies in a single center of Korea: origination, course, and termination anomalies of aberrant coronary arteries detected by ECG-gated cardiac MDCT. BMC Cardiovasc Disord 2014; 14: 48.

18. Zeina AR, Blinder J, Sharif D, et al. Congenital coronary artery anomalies in adults: non-invasive assessment with multidetector CT. Br J Radiol 2009; 82: 254-261.

19. Koşar P, Ergun E, Öztürk C, Koşar U. Anatomic variations and anomalies of the coronary arteries: 64-slice CT angiographic appearance. Diagn Interv Radiol 2009; 15: 275-283.

20. Hacioglu Y, Budoff M. Is the left anterior descending artery really absent? - a decisive input from coronary CT angiography. Catheter Cardiovasc Interv 2010; 76: 117-120.

21. Cho SH, Joo HC, Yoo KJ, Youn YN. Anomalous origin of right coronary artery from left coronary sinus: surgical management and clinical result. Thorac Cardiovasc Surg 2015; 63: 360-366. 
22. Click RL, Holmes DR Jr, Vliestra RE, et al. Anomalous coronary arteries: location, degree of atherosclerosis and effect on survivala report from the coronary artery surgery study. J Am Coll Radiol 1989; 13: 531-537.

23. Dodd JD, Ferencik M, Liberthson RR, et al. Congenital anomalies of coronary artery origin in adults: 64-MDCT appearance. AJR 2007; 188: 138-146.

24. Taylor AJ, Rogan KM, Virmani R. Sudden cardiac death associated with isolated congenital artery anomalies. J Am Coll Radiol 1992; 20: 640-647.

25. Krupiński M, Urbańczyk-Zawadzka M, Laskowicz B, et al. Anomalous origin of the coronary artery from the wrong coronary sinus evaluated with computed tomography: "High-risk" anatomy and its clinical relevance. Eur Radiol 2014; 24: 2353-2359.

26. Desmet W, Vanhaecke J, Vrolix M, et al. Isolated single coronary artery: a review of 50,000 consecutive coronary angiographies. Eur Heart J 1992; 13: 1637-1640.

27. Desmet W, Vanhaecke J, Vrolix M, et al. Isolated single coronary artery: a review of 50,000 consecutive coronary angiographies. Eur Heart J 1992; 13: 1637-1640.

28. Aldana-Sepulveda N, Restrepo CS, Kimura-Hayama E. Single coronary artery: spectrum of imaging findings with multidetector CT. J Cardiovasc Comput Tomogr 2013; 7: 391-399.
29. Patel KB, Gupta H, Nath H, et al. Origin of all three major coronary arteries from the right sinus of Valsalva: clinical, angiographic, and magnetic resonance imaging findings and incidence in a select referral population. Catheter Cardiovasc Interv 2007; 69: 711-718.

30. Alegria JR, Herrmann J, Holmes DR, Jr, et al. Myocardial bridging. Eur Heart J 2005; 26: 1159-1168.

31. Nakanishi R, Rajani R, Ishikawa Y, et al. Myocardial bridging on coronary CTA: an innocent bystander or a culprit in myocardial infarction? J Cardiovasc Comput Tomogr 2012; 6: 3-13.

32. Manghat NE, Morgan-Hughes JG, Marshall AJ, Roobottom CA. Multidetector row computed tomography: imaging congenital coronary artery anomalies in adults. Heart 2005; 91: 1515-1522.

33. Armsby L, Keane J, Sherwood M, et al. Management of coronary artery fistulae patient selection and results of transcatheter closure. J Am Coll Cardiol 2002; 39: 1026-1032.

34. Zhou CS, Zhang LJ, Wang YN, et al. Incidence and classification diagnosis of coronary artery fistula at dual-source CT coronary angiography. Zhonghua Yi Xue Za Zhi 2012; 92: 3336-3340.

35. Zenooz NA, Habibi R, Mammen L, et al. Coronary artery fistulas: CT findings. Radiographics 2009; 29: 781-789. 